\title{
A Proposed Evidence-Based Local Guideline for Definition of Multidrug- Resistant (MDR), Extensively Drug-Resistant (XDR) and Pan Drug- Resistant (PDR) Bacteria by the Microbiology Laboratory
}

\author{
Arash Eatemadi' ${ }^{1}$, Elham Al Risi ${ }^{2}$, Amit Kasilas Kasliwal ${ }^{3}$, Ahmed Al Za'abi ${ }^{4}$, Hamid Moradzadegan, \\ Zahra Aslani' \\ ${ }^{1}$ Division of Infectious Diseases, General Medicine Department, Suhar hospital, Suhar, Sultanate of Oman \\ ${ }^{2}$ Pathology and blood bank Department, Suhar hospital, Suhar, Sultanate of Oman \\ ${ }^{3}$ Division of Acute Medicine, General Medicine Department, Suhar hospital, Suhar, Sultanate of Oman \\ ${ }^{4}$ Division of Clinical Pharmacy, Pharmacy Department, Suhar hospital, Suhar, Sultanate of Oman \\ ${ }^{5}$ Laboratory and Blood bank Department, Arvand hospital, Ahwaz, Iran \\ ${ }^{6}$ Division of Infectious Diseases, Internal Medicine Department, Milad hospital, Ahwaz, Iran
}

\begin{abstract}
Multi-drug resistant organisms (MDROs) are defined as microorganisms, predominantly bacteria, that are resistant to one or more classes of antimicrobial agents. These pathogens are frequently resistant to most available antimicrobial agents and deserve special attention in healthcare facilities. Generally, MDRO infections have clinical manifestations similar to infections caused by susceptible pathogens. Despite of two different classifications by WHO and CDC, still there are debates about update definition of MDROs in medical literature. Here we provide an updated local guideline for definitions of various MDROs by microbiology laboratory.
\end{abstract}

KEY WORDS: Multi-drug resistant organisms (MDROs), Antimicrobial agent, Healthcare facilities, microbiology laboratory

Worldwide emerging data suggest that multidrug resistant organisms (MDROs) have considerably increased in the recent decades. The worldwide spread of organisms that are resistant to multiple antibiotics, generally referred to as multidrug-resistant organisms (MDRO), has become a public health concern. $(1,2)$ Although the names of certain multi-drug resistant organisms (MDROs) describe resistance to only one agent as in methicillin-resistant staphylococcus aureus (MRSA) and vancomycin resistant enterococcus (VRE), these pathogens are frequently resistant to most available antimicrobial agents. These highly resistant organisms deserve special attention in healthcare facilities. In addition to MRSA and VRE, certain gram- negative bacteria (GNB), including extended spectrum beta-lactamases- producing Enterobacteriaceae (ESBL-PE), Carbapenem-resistant Pseudomonas aeruginosa \& Acinetobacter baumannii (CRPA \& CRAB), other carbapenem-resistant Enterobacteriaceae (CRE), also Stenotrophomonas maltophilia and Burkholderia cepacia that are intrinsically resistant to the broadest-spectrum antimicrobial agents are now emerging worldwide. In most instances, MDRO infections have clinical manifestations that are similar to infections caused by susceptible pathogens. However, options for treating patients with these infections are usually limited. Increased lengths of stay, costs, and mortality also have been associated with MDROs. Among MDROs, MRSA is differently from other MDROs. When patients with MRSA have been compared to patients with methicillin-susceptible S. aureus (MSSA), MRSA-colonized patients more frequently develop symptomatic infections. Furthermore, higher case fatality rates have been observed for certain MRSA infections, including bacteremia. Staphylococcus aureus, vancomycin-intermediate (VISA) and resistant (VRSA) are also difficult to treat emerging variants.

The main sources of information on rates of infection caused by MDROs come from regional, national or international surveillance systems, published epidemiological studies and outbreak reports. On 2017, WHO published its first list of antibiotic-resistant "priority pathogens" (12 families of bacteria that pose the greatest threat to human health). (3) The criteria for selecting pathogens on the list were:

1- How deadly the infections they cause are; whether their treatment requires long hospital stays

2- How frequently they are resistant to existing antibiotics when people in communities catch them

3- How easily they spread between animals, from animals to humans, and from person to person 


\section{International Journal of Current Science Research and Review}

ISSN: 2581-8341

Volume 04 Issue 03 March 2021

DOI: 10.47191/ijcsrr/V4-i3-01, Impact Factor: 5.825

IJCSRR @ 2021

www.ijcsrr.org

4- Whether they can be prevented (e.g through good hygiene and vaccination)

5- How many treatment options remain

6- Whether new antibiotics to treat them are already in the R\&D pipeline.

The list highlights in particular the threat of gram-negative bacteria that are resistant to multiple antibiotics. The WHO list is divided into three categories according to the urgency of need for new antibiotics: critical, high and medium priority. (3) (Table 1)

The most critical group of all includes multidrug resistant bacteria that pose a particular threat in hospitals, nursing homes, and among patients whose care requires devices such as ventilators and blood catheters. They include Acinetobacter, Pseudomonas and various Enterobacteriaceae (including Klebsiella, E. coli, Serratia, and Proteus). They can cause severe and often deadly infections such as bloodstream infections and pneumonia. These bacteria have become resistant to a large number of antibiotics, including carbapenems and third generation cephalosporins - the best available antibiotics for treating multi-drug resistant bacteria. The second and third tiers in the list - the high and medium priority categories - contain other increasingly drug-resistant bacteria that cause more common diseases such as gonorrhea and food poisoning caused by salmonella.

Tuberculosis was not included in the list. Other bacteria that were not included, such as streptococcus A and B and chlamydia, have low levels of resistance to existing treatments and do not currently pose a significant public health threat.

On the other hand, CDC is also concerned about rising resistant infections in the community, which can put more people at risk, make spread more difficult to identify and contain, and threaten the progress made to protect patients in healthcare. The emergence and spread of new forms of resistance remains a concern. On 2019, CDC released the lists of 18 antibiotic-resistant bacteria and fungi into three categories based on level of concern to human health-urgent, serious and concerning. (4) The report also includes a Watch List with three threats that have not spread resistance widely in the U.S. but could become common without a continued aggressive approach. (4) (Table 2)

A resistant interpretation of an isolate can be determined using disk diffusion, broth microdilution or agar dilution following Clinical and Laboratory Standards Institute (CLSI) guidelines for susceptibility testing and interpretation of Enterobacteriaceae, P. aeruginosa and Acinetobacter spp. (5) Current CLSI M100 breakpoints should be used to determine antimicrobial susceptibility of isolates (5). MIC or zone diameter value breakpoints and interpretive categories are established per CLSI document M235 for categories of susceptible, intermediate, and resistant (and susceptible-dose dependent and non-susceptible, when appropriate).

$\square$ Susceptible (S) - a category defined by a breakpoint that implies that isolates with an MIC at or below or a zone diameter at or above the susceptible breakpoint are inhibited by the usually achievable concentrations of antimicrobial agent when the dosage recommended to treat the site of infection is used, resulting in likely clinical efficacy.

$\square$ Susceptible-dose dependent (SDD) - a category defined by a breakpoint that implies that susceptibility of an isolate depends on the dosage regimen that is used in the patient. To achieve levels that are likely to be clinically effective against isolates for which the susceptibility testing results (either MICs or zone diameters) are in the SDD category, it is necessary to use a dosage regimen (ie, higher doses, more frequent doses, or both) that results in higher drug exposure than that achieved with the dose that was used to establish the susceptible breakpoint. Consideration should be given to the maximum, literature-supported dosage regimen, because higher exposure gives the highest probability of adequate coverage of an SDD isolate. Appendix E lists the doses used when establishing SDD categories. The drug label should be consulted for recommended doses and adjustment for organ function. (NOTE: The SDD category may be assigned when doses well above those used to calculate the susceptible breakpoint are supported by the literature, widely used clinically, and/or approved and for which sufficient data to justify the designation exist and have been reviewed.)

Intermediate (I) - a category defined by a breakpoint that includes isolates with MICs or zone diameters within the intermediate range that approach usually attainable blood and tissue levels and/or for which response rates may be lower than for susceptible isolates. (NOTE: The intermediate category implies clinical efficacy in anatomical sites where the drugs are physiologically concentrated.)

Resistant (R) - a category defined by a breakpoint that implies that isolates with an MIC at or above or a zone diameter at or below the resistant breakpoint are not inhibited by the usually achievable concentrations of the agent with normal dosage schedules 


\section{International Journal of Current Science Research and Review}

ISSN: 2581-8341

Volume 04 Issue 03 March 2021

DOI: 10.47191/ijcsrr/V4-i3-01, Impact Factor: 5.825

IJCSRR@ 2021

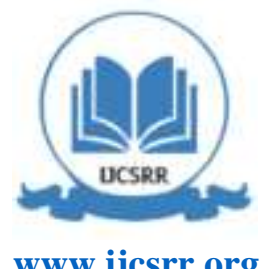

www.ijcsrr.org

and/or that demonstrate MICs or zone diameters that fall in the range in which specific microbial resistance mechanisms are likely, and clinical efficacy of the agent against the isolate has not been reliably shown in treatment studies.

$\square$ Non-susceptible (NS) - a category used for isolates for which only a susceptible breakpoint is designated because of the absence
or rare occurrence of resistant strains. Isolates for which the antimicrobial agent MICs are above or the zone diameters are below
the value indicated for the susceptible breakpoint should be reported as non-susceptible. (NOTE 1: An isolate that is interpreted as
Non-susceptible does not necessarily mean that the isolate has a resistance mechanism. It is possible that isolates with MICs above
the susceptible breakpoint that lacks resistance mechanisms may be encountered within the wild-type distribution after the time the
susceptible-only breakpoint was set; NOTE 2: The term "non-susceptible” should not be used when the text is describing an
organism/drug category with intermediate and resistant interpretive categories.)
Some laboratories may routinely use other breakpoint interpretations such as European Committee on Antimicrobial Susceptibility
Testing (EUCAST) that differ from CLSI recommendations. Laboratories using non-CLSI breakpoints, should disclose this
information in their reports to provincial public health laboratories. Certain species of Enterobacteriaceae should not be tested for
particular antimicrobial agents because of intrinsic resistance. (for example, Proteus spp. are intrinsically resistant to tigecycline and
colistin while Serratia spp. are naturally resistant to Colistin)
On the other hand, recently EUCAST has changed the definitions of susceptibility testing categories S, I and R: (6)

S - Susceptible: A microorganism is categorized as "Susceptible, standard dosing regimen", when there is a high likelihood of therapeutic success using a standard dosing regimen of the agent.

I - Susceptible, increased exposure: A microorganism is categorized as "Susceptible, Increased exposure when there is a high likelihood of therapeutic success because exposure to the agent is increased by adjusting the dosing regimen or by its concentration at the site of infection.

R - Resistant: A microorganism is categorized as "Resistant" when there is a high likelihood of therapeutic failure even when there is increased exposure.

Despite of these two different classifications by WHO and CDC, still there are debates about update definition of MDROs in medical literature. Wrong or old definitions for MDROs, especially regarding GNBs, will be misleading and can cause false epidemiologic statistics and clinical decision making. CDC typically uses MDRO to refer to an isolate that is resistant to at least one antibiotic in three or more drug classes. (4)

According to Canadian recommendations for laboratory interpretation of multiple or extensive drug resistance in clinical isolates of Enterobacteriaceae, Acinetobacter species and Pseudomonas aeruginosa there is only definition for MDR Enterobacteriaceae and XDR Acinetobacter spp. or P. aeruginosa definition. (7) Regarding Enterobacteriaceae, an isolate should be considered a MDRO if it is resistant to THREE OR FOUR of the SIX antimicrobial groups including Tobramycin OR gentamicin (except for Serratia spp.), Piperacillin-tazobactam, Imipenem OR meropenem (except for Proteus spp.), Cefotaxime OR ceftriaxone OR ceftazidime, Ciprofloxacin and Trimethoprim-sulfamethoxazole. However, about Acinetobacter spp. or P. aeruginosa, there are no final recommendations for MDRO definitions for Acinetobacter spp. or P. aeruginosa. The previous interim recommendations for Acinetobacter spp. or P. aeruginosa MDRO status should be disregarded at this time (8). An isolate should be considered an XDRO if it is resistant to All of the five antimicrobial groups including Ciprofloxacin, Piperacillin-tazobactam, Ceftazidime, Imipenem or meropenem and Tobramycin. The most important pitfall of this classification is lack of definition for MDR Acinetobacter spp. or P. aeruginosa. Also, they have no idea about XDR and PDR pathogens.

We preferred to define MDR, XDR and PDR according to the "international expert proposal for interim standard definitions for acquired resistance" (9). MDR refers to strains that exhibit resistance to more than three or more antimicrobial drug classes $(9,10)$. XDR refers to strains resistant to all but two drug classes $(9,11)$. Pan-drug resistance refers to resistance exhibited by the strains to all drug classes $(9,12)$ Thus, we reviewed all new studies regarding definition and reporting of MDROs (13-24) and have created an updated, local guideline for definition for MDR, XDR and PDR pathogens which is scientific and practical for all microbiology laboratories at national level. (Table 3) 


\section{International Journal of Current Science Research and Review}

ISSN: 2581-8341

Volume 04 Issue 03 March 2021

DOI: 10.47191/ijesrr/V4-i3-01, Impact Factor: 5.825

IJCSRR@ 2021

Www.ijcsrr.org

In conclusion, although till date there was no comprehensive consensus regarding detailed definitions of MDR, XDR and PDR pathogens have been proposed, however, our new practical definition according to latest evidences can help microbiology laboratories for report and infectious disease physicians to deal better with MDROs in practice.

Table 1: WHO priority pathogens list

\begin{tabular}{|l|l|l|}
\hline Priority 1: CRITICAL & Priority 2: HIGH & Priority 3: MEDIUM \\
\hline *Carbapenem-resistant & *vancomycin resistant enterococcus & *Streptococcus pneumoniae, \\
Acinetobacter baumannii & *Methicillin-resistant, vancomycin intermediate \& & penicillin-non-susceptible \\
*Carbapenem-resistant & vancomycin resistant Staphylococcus aureus & *Hemophilus influenzae, \\
Pseudomonas aeruginosa & $*$ Helicobacter pylori, clarithromycin-resistant & ampicillin-resistant \\
$*$ Carbapenem-resistant & $*$ Campylobacter spp., fluoroquinolone-resistant & *Shigella spp., fluoroquinolone- \\
Enterobacteriaceae & *Salmonellae, fluoroquinolone-resistant & resistant \\
$*$ ESBL-Producing & *Neisseria gonorrhoeae, cephalosporin-resistant, & \\
Enterobacteriaceae & fluoroquinolone-resistant & \\
\hline
\end{tabular}

Table 2: CDC 2019 ANTIBIOTIC Resistance (AR) Threats Report

\begin{tabular}{|c|c|c|c|}
\hline Urgent Threats & Serious Threats & Concerning Threats & Watch List \\
\hline $\begin{array}{l}\text { *Carbapenem-resistant } \\
\text { Acinetobacter baumannii } \\
\text { *Candida auris } \\
\text { *C. difficile } \\
\text { *Carbapenem-resistant } \\
\text { Enterobacteriaceae } \\
\text { *Drug-resistant Neisseria } \\
\text { gonorrhoeae }\end{array}$ & $\begin{array}{l}\text { *Drug-resistant Campylobacter } \\
\text { *Drug-resistant Candida } \\
\text { *ESBL- Producing } \\
\text { Enterobacteriaceae } \\
\text { *Vancomycin resistant } \\
\text { enterococcus } \\
\text { *MDR P. aeruginosa } \\
\text { *Drug-resistant S. Typhi } \\
\text { \& nontyphoidal Salmonella } \\
\text { *Drug-resistant Shigella } \\
\text { *Methicillin-resistant } \\
\text { Staphylococcus aureus } \\
\text { *Drug-resistant S. pneumoniae } \\
\text { *Drug-resistant Tuberculosis }\end{array}$ & $\begin{array}{l}\text { *Erythromycin-Resistant } \\
\text { Group A Streptococcus } \\
\text { *Clindamycin-resistant } \\
\text { Group B Streptococcus }\end{array}$ & $\begin{array}{l}\text { *Azole-resistant } \\
\text { Aspergillus fumigatus } \\
\text { *Drug-resistant } \\
\text { Mycoplasma genitalium } \\
\text { *Drug-resistant } \\
\text { Bordetella pertussis }\end{array}$ \\
\hline
\end{tabular}

Table 3: Proposed new definition of MDR, XDR, PDR

\begin{tabular}{|c|c|c|c|c|}
\hline Pathogen(s) & Must be tested & MDR & XDR & PDR \\
\hline Staphylococcus aureus & $\begin{array}{l}\text { *Gentamicin } \\
\text { *Oxacillin } \\
\text { *Ciprofloxacin } \\
\text { *TMP/SMX } \\
\text { *Vancomycin or Teicoplanin } \\
\text { *Tigecycline } \\
\text { *Erythromycin } \\
\text { *Clindamycin } \\
\text { *Linezolid } \\
\text { *Doxycycline or Minocycline } \\
\text { * Rifampicin }\end{array}$ & $\begin{array}{l}\text { non- } \\
\text { susceptible } \\
\text { to } \geq 1 \text { agent } \\
\text { in } \geq 3 \\
\text { antimicrobial } \\
\text { categories. }\end{array}$ & $\begin{array}{l}\text { non- } \\
\text { susceptible } \\
\text { to } \geq 1 \text { agent } \\
\text { in all but } \leq 2 \\
\text { categories. } \\
\text { (Only } \\
\text { susceptible } \\
\text { to } \quad 1-2 \\
\text { remaining } \\
\text { agent) }\end{array}$ & $\begin{array}{l}\text { non- } \\
\text { susceptible } \\
\text { to all } \\
\text { antimicrobial } \\
\text { agents listed. }\end{array}$ \\
\hline
\end{tabular}


International Journal of Current Science Research and Review

ISSN: 2581-8341

Volume 04 Issue 03 March 2021

DOI: 10.47191/ijcsrr/V4-i3-01, Impact Factor: 5.825

IJCSRR @ 2021

www.ijcsrr.org

\begin{tabular}{|c|c|c|c|c|c|}
\hline Enterococcus faecalis & $\begin{array}{l}\text { *Gentamicin \& Streptomycin (high level) } \\
\text { *Imipenem or Meropenem } \\
\text { *Vancomycin or Teicoplanin } \\
\text { *Tigecycline } \\
\text { *Ampicillin } \\
\text { *Linezolid } \\
\text { *Doxycycline } \\
\text { *Ciprofloxacin }\end{array}$ & $\begin{array}{l}\text { Same as } \\
\text { above }\end{array}$ & $\begin{array}{l}\text { Same as } \\
\text { above }\end{array}$ & $\begin{array}{l}\text { Same } \\
\text { above }\end{array}$ & \\
\hline Enterococcus faecium & $\begin{array}{l}\text { *Gentamicin \& Streptomycin } \\
\text { *Vancomycin or Teicoplanin } \\
\text { *Tigecycline (when no other option) } \\
\text { *Ampicillin } \\
\text { *Linezolid } \\
\text { *Doxycycline } \\
\text { *Ciprofloxacin }\end{array}$ & $\begin{array}{l}\text { Same } \\
\text { above }\end{array}$ & $\begin{array}{l}\text { Same as } \\
\text { above }\end{array}$ & $\begin{array}{l}\text { Same } \\
\text { above }\end{array}$ & as \\
\hline E. coli & $\begin{array}{l}\text { *Amoxicillin-clavulanate } \\
\text { *Amikacin or Gentamycin } \\
\text { *Piperacillin/tazobactam } \\
\text { *Imipenem or Meropenem } \\
\text { *Cefazolin or Cefuroxime } \\
\text { *Cefotaxime or Ceftriaxone or Ceftazidime } \\
\text { *Ciprofloxacin } \\
\text { *Trimethoprim-sulfamethoxazole } \\
\text { *Doxycycline } \\
\text { *Tigecycline (when no other option) } \\
\text { *Colistin (when no other option) }\end{array}$ & $\begin{array}{l}\text { Same } \\
\text { above }\end{array}$ & $\begin{array}{l}\text { Same } \\
\text { above }\end{array}$ & $\begin{array}{l}\text { Same } \\
\text { above }\end{array}$ & as \\
\hline Klebsiella spp. & $\begin{array}{l}\text { *Amoxicillin-clavulanate } \\
\text { *Amikacin or Gentamycin } \\
\text { *piperacillin/tazobactam } \\
\text { *Imipenem or Meropenem } \\
\text { *Cefazolin or Cefuroxime } \\
\text { *Cefotaxime or Ceftriaxone or Ceftazidime } \\
\text { *Ciprofloxacin } \\
\text { *Trimethoprim-sulfamethoxazole } \\
\text { *Doxycycline } \\
\text { *Tigecycline (when no other option) } \\
\text { *Colistin (when no other option) }\end{array}$ & $\begin{array}{l}\text { Same } \\
\text { above }\end{array}$ & $\begin{array}{l}\text { Same } \\
\text { above }\end{array}$ & $\begin{array}{l}\text { Same } \\
\text { above }\end{array}$ & as \\
\hline $\begin{array}{l}\text { Providencia spp. } \\
\text { *intrinsically resistant to } \\
\text { Colistin \& Tigecycline }\end{array}$ & $\begin{array}{l}\text { *Amikacin } \\
\text { *piperacillin-tazobactam } \\
\text { *Imipenem or Meropenem } \\
\text { *Cefuroxime } \\
\text { *Cefotaxime or Ceftriaxone or Ceftazidime } \\
\text { *Ciprofloxacin } \\
\text { *Trimethoprim-sulfamethoxazole }\end{array}$ & $\begin{array}{l}\text { Same } \\
\text { above }\end{array}$ & $\begin{array}{l}\text { Same } \\
\text { above }\end{array}$ & $\begin{array}{l}\text { Same } \\
\text { above }\end{array}$ & as \\
\hline $\begin{array}{l}\text { Citrobacter spp. } \\
\text { (Freundi, Koseri,...) }\end{array}$ & $\begin{array}{l}\text { *Amikacin or Gentamycin } \\
\text { *piperacillin-tazobactam } \\
\text { *Imipenem or Meropenem }\end{array}$ & $\begin{array}{l}\text { Same as } \\
\text { above }\end{array}$ & $\begin{array}{l}\text { Same as } \\
\text { above }\end{array}$ & $\begin{array}{l}\text { Same } \\
\text { above }\end{array}$ & \\
\hline
\end{tabular}


ISSN: 2581-8341

Volume 04 Issue 03 March 2021

DOI: 10.47191/ijcsrr/V4-i3-01, Impact Factor: 5.825

IJCSRR @ 2021

www.ijcsrr.org

\begin{tabular}{|c|c|c|c|c|c|c|}
\hline & $\begin{array}{l}\text { *Cefuroxime } \\
* \text { Cefotaxime or Ceftriaxone or Ceftazidime } \\
\text { *Ciprofloxacin } \\
\text { *Trimethoprim-sulfamethoxazole } \\
\text { *Doxycycline } \\
\text { *Tigecycline (when no other option) } \\
\text { *Colistin (when no other option) }\end{array}$ & & & & & \\
\hline $\begin{array}{l}\text { Enterobacter aerogenes } \\
\& \\
\text { E. cloacae }\end{array}$ & $\begin{array}{l}\text { *Amikacin or Gentamycin } \\
\text { *piperacillin-tazobactam } \\
\text { *Imipenem or Meropenem } \\
* \text { Cefuroxime } \\
* \text { Cefotaxime or Ceftriaxone or Ceftazidime } \\
\text { *Ciprofloxacin } \\
\text { *Trimethoprim-sulfamethoxazole } \\
\text { *Doxycycline } \\
\text { *Tigecycline (when no other option) } \\
* \text { Colistin (when no other option) }\end{array}$ & $\begin{array}{l}\text { Same as } \\
\text { above }\end{array}$ & $\begin{array}{l}\text { Same } \\
\text { above }\end{array}$ & as & $\begin{array}{l}\text { Same } \\
\text { above }\end{array}$ & \\
\hline $\begin{array}{l}\text { Morganella morganii } \\
\text { *intrinsically resistant to } \\
\text { colistin \& tigecycline }\end{array}$ & $\begin{array}{l}\text { *Amikacin or Gentamycin } \\
\text { *piperacillin-tazobactam } \\
\text { *Imipenem or Meropenem } \\
\text { *Cefotaxime or Ceftriaxone or Ceftazidime } \\
\text { *Ciprofloxacin } \\
\text { *Trimethoprim-sulfamethoxazole }\end{array}$ & $\begin{array}{l}\text { Same as } \\
\text { above }\end{array}$ & $\begin{array}{l}\text { Same } \\
\text { above }\end{array}$ & as & $\begin{array}{l}\text { Same } \\
\text { above }\end{array}$ & \\
\hline $\begin{array}{l}\text { Serratia marcescens } \\
\text { *intrinsically resistant to } \\
\text { colistin }\end{array}$ & $\begin{array}{l}\text { *Amikacin or Gentamycin } \\
\text { *piperacillin-tazobactam } \\
\text { *Imipenem or Meropenem } \\
\text { *Cefotaxime or Ceftriaxone or Ceftazidime } \\
\text { *Ciprofloxacin or Levofloxacin } \\
\text { *Trimethoprim-sulfamethoxazole } \\
\text { *Tigecycline (when no other option) }\end{array}$ & $\begin{array}{l}\text { Same } \\
\text { above }\end{array}$ & $\begin{array}{l}\text { Same } \\
\text { above }\end{array}$ & as & $\begin{array}{l}\text { Same } \\
\text { above }\end{array}$ & \\
\hline $\begin{array}{l}\text { Non Mirabilis Proteus } \\
\text { (Vulgaris, Penneri,...) } \\
\text { *intrinsically resistant to } \\
\text { colistin \& tigecycline }\end{array}$ & $\begin{array}{l}\text { *Amoxicillin-clavulanate } \\
\text { *Amikacin or Gentamycin } \\
\text { *piperacillin-tazobactam } \\
\text { *Imipenem or Meropenem } \\
\text { *Cefotaxime or Ceftriaxone or Ceftazidime } \\
\text { *Ciprofloxacin } \\
\text { *Trimethoprim-sulfamethoxazole }\end{array}$ & $\begin{array}{l}\text { Same } \\
\text { above }\end{array}$ & $\begin{array}{l}\text { Same } \\
\text { above }\end{array}$ & as & $\begin{array}{l}\text { Same } \\
\text { above }\end{array}$ & \\
\hline $\begin{array}{l}\text { Proteus Mirabilis } \\
\text { *intrinsically resistant to } \\
\text { colistin \& tigecycline }\end{array}$ & $\begin{array}{l}\text { *Amoxicillin-clavulanate } \\
\text { *Amikacin or Gentamycin } \\
\text { *piperacillin-tazobactam } \\
\text { *Imipenem or Meropenem } \\
\text { *Cefazolin or Cefuroxime } \\
\text { *Cefotaxime or Ceftriaxone or Ceftazidime } \\
\text { *Ciprofloxacin or Levofloxacin } \\
\text { *Trimethoprim-sulfamethoxazole }\end{array}$ & $\begin{array}{l}\text { Same } \\
\text { above }\end{array}$ & $\begin{array}{l}\text { Same } \\
\text { above }\end{array}$ & as & $\begin{array}{l}\text { Same } \\
\text { above }\end{array}$ & \\
\hline
\end{tabular}




\section{International Journal of Current Science Research and Review}

ISSN: 2581-8341

Volume 04 Issue 03 March 2021

DOI: 10.47191/ijesrr/V4-i3-01, Impact Factor: 5.825

IJCSRR@ 2021

www.ijcsrr.org

\begin{tabular}{|c|c|c|c|c|c|}
\hline $\begin{array}{l}\text { Pseudomonas } \\
\text { aeruginosa }\end{array}$ & $\begin{array}{l}\text { *Amikacin or Gentamycin } \\
\text { *piperacillin-tazobactam } \\
\text { *Imipenem or Meropenem } \\
\text { *Ceftazidime or Cefepime } \\
\text { *Ciprofloxacin or Levofloxacin } \\
* \text { Colistin (when no other option) }\end{array}$ & $\begin{array}{l}\text { Same as } \\
\text { above }\end{array}$ & $\begin{array}{l}\text { Same as } \\
\text { above }\end{array}$ & $\begin{array}{l}\text { Same } \\
\text { above }\end{array}$ & \\
\hline $\begin{array}{l}\text { Acinetobacter } \\
\text { (including } \\
\text { A. baumannii) }\end{array}$ & $\begin{array}{l}\text { *Amikacin or Gentamycin } \\
\text { *Imipenem or Meropenem } \\
\text { *Cefotaxime or Ceftriaxone or Ceftazidime } \\
\text { *Ciprofloxacin or Levofloxacin } \\
\text { *Trimethoprim-sulfamethoxazole } \\
\text { *Doxycycline or Minocycline } \\
\text { *Tigecycline (when no other option) } \\
\text { *Colistin (when no other option) }\end{array}$ & $\begin{array}{l}\text { Same as } \\
\text { above }\end{array}$ & $\begin{array}{l}\text { Same as } \\
\text { above }\end{array}$ & $\begin{array}{l}\text { Same } \\
\text { above }\end{array}$ & \\
\hline $\begin{array}{l}\text { Stenotrophomonas } \\
\text { maltophilia }\end{array}$ & $\begin{array}{l}\text { *Ceftazidime } \\
\text { *Levofloxacin } \\
\text { *Trimethoprim-sulfamethoxazole } \\
\text { *Tigecycline (when no other option) } \\
\text { *Colistin (when no other option) } \\
\text { *Minocycline or Doxycycline }\end{array}$ & & & & \\
\hline
\end{tabular}

\section{REFERENCES}

1. European Commission Action plan against the rising threats from antimicrobial resistance: Communication from the Commission to the European Parliament and the Council. 15 Brussels, 2011.

2. The bacterial challenge: time to react. ECDC/EMEA joint technical report. EMEA Doc. Ref. EMEA/576176/2009. Stockholm, September 2009. ISBN 978-92-9193-193-4 Available:

https://ecdc.europa.eu/sites/portal/files/media/en/publications/Publications/0909_TER_The_Bacterial_Challenge_Time_to _React.pdf [Accessed March 2019].

3. https://www.who.int/news/item/27-02-2017-who-publishes-list-of-bacteria-for-which-new-antibiotics-are-urgently-needed

4. https://www.cdc.gov

5. https://www.clsi.org

6. https://www.eucast.org

7. German GJ, Gilmour M, Tipples G, et al. Canadian recommendations for laboratory interpretation of multiple or extensive drug resistance in clinical isolates of Enterobacteriaceae, Acinetobacter species and Pseudomonas aeruginosa. Can Commun Dis Rep. 2018;44(1):29-34. Published 2018 Jan 4. doi:10.14745/ccdr. v44i01a07

8. German GJ, Jamieson FB, Gilmour M, Almohri H, Bullard J, Domingo MC et al. Interim recommendations for the reporting of extensively drug resistant and pan-drug resistant isolates of Enterobacteriaceae, Pseudomonas aeruginosa, Acinetobacter spp. and Stenotrophomonas maltophilia. Can Commun Dis Rep 2016;42(4):91-7. Available from:

https://www.canada.ca/en/public-health/services/reports-publications/canada-communicable-disease-report-ccdr/monthlyissue/2016-42/ccdr-volume-42-4-april-7-2016/ccdr-volume-42-4-april-7-2016-vaccine-preventable-diseases-3.html

9. Magiorakos AP, Srinivasan A, Carey RB, Carmeli Y, Falagas ME, Giske CG. et al. Multidrug-resistant, extensively drugresistant and pandrug-resistant bacteria: an international expert proposal for interim standard definitions for acquired resistance. Clin Microbiol Infect. 2012; 18:268-281.

10. Leski TA, Vora GJ, Barrows BR, Piemental G, House BL, Nicklasson M. et al. Molecular Characterization of Multidrug Resistant Hospital Isolates Using the Antimicrobial Resistance Determinant Microarray. PLoS One. 2013;8: e69507.

11. Levy SB, Marshall B. Antibacterial resistance worldwide: causes, challenges and responses. Nat Med. 2004; 10: S122-9.

12. Lee CM, Lim HK, Liu CP, Tseng HK. Treatment of pan drug resistant Acinetobacter. Scand J Infect Dis. 2005; 37:195-9. 


\section{International Journal of Current Science Research and Review}

ISSN: 2581-8341

Volume 04 Issue 03 March 2021

DOI: 10.47191/ijcsrr/V4-i3-01, Impact Factor: 5.825

IJCSRR @ 2021

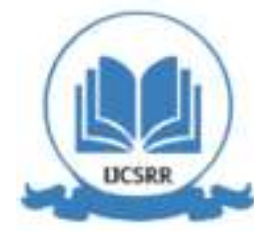

www.ijcsrr.org

13. Gould IM, Wang GQ, Wu JJ, Lim VK, Hutchinson J, Walsh T, Turnidge J. MDRO Beijing Consensus Meeting Report: Global burden of multidrug-resistant organisms' current antimicrobial resistance problems in Asia-Pacific. J Glob Antimicrob Resist. 2014 Mar;2(1):7-9. doi: 10.1016/j.jgar.2013.10.005. Epub 2013 Nov 8. PMID: 27873642.

14. Anaya-Baz B, Maldonado N, Palacios-Baena ZR, Palomo V, Pezzani MD, Chiesi S, Razzaboni E, Compri M, Tacconelli E, Rodriguez-Baño J. Systematic literature review of the burden and outcomes of infections due to multidrug-resistant organisms in Europe: the ABOUT-MDRO project protocol. BMJ Open. 2020 May 5;10(5): e030608. doi: 10.1136/bmjopen2019-030608. PMID: 32371505; PMCID: PMC7228488.

15. Sun W, Hesse S, Xu M, Childs RW, Zheng W, Williamson PR. "Real-Time" High-Throughput Drug and Synergy Testing for Multidrug-Resistant Bacterial Infection: A Case Report. Front Med (Lausanne). 2018 Sep 20;5: 267. doi: 10.3389/fmed.2018.00267. PMID: 30298132; PMCID: PMC6160733.

16. Burnham JP, Kwon JH, Olsen MA, Babcock HM, Kollef MH. Readmissions with Multidrug-Resistant Infection in Patients with Prior Multidrug Resistant Infection. Infect Control Hosp Epidemiol. 2018 Jan;39(1):12-19. doi: 10.1017/ice.2017.254. Epub 2017 Dec 17. PMID: 29248023; PMCID: PMC6233291.

17. Kalluru S, Eggers S, Barker A, Shirley D, Sethi AK, Sengupta S, Yeptho K, Safdar N. Risk factors for infection with multidrug-resistant organisms in Haryana, India. Am J Infect Control. 2018 Mar;46(3):341-345. doi: 10.1016/j.ajic.2017.08.021. Epub 2017 Nov 1. PMID: 29102426; PMCID: PMC6055232.

18. Spachmann PJ, May M, Vetterlein MW, Fritsche HM, Steffen S, Schostak M, Wagenlehner FM, Burger M, Weylandt KH, Salzberger B, Brookman-May SD, Gilfrich C; MR2 study group. Awareness and perception of multidrug-resistant organisms and antimicrobial therapy among internists vs. surgeons of different specialties: Results from the German MR2 Survey. Caspian J Intern Med. 2019 Spring;10(2):132-141. doi: 10.22088/cjim.10.2.132. PMID: 31363391; PMCID: PMC6619476.

19. Han JH, Lapp Z, Bushman F, Lautenbach E, Goldstein EJC, Mattei L, Hofstaedter CE, Kim D, Nachamkin I, Garrigan C, Jain T, Bilker W, Wolford HM, Slayton RB, Wise J, Tolomeo P, Snitkin ES. Whole-Genome Sequencing to Identify Drivers of Carbapenem-Resistant Klebsiella pneumoniae Transmission within and between Regional Long-Term Acute-Care Hospitals. Antimicrob Agents Chemother. 2019 Oct 22;63(11): e01622-19. doi: 10.1128/AAC.01622-19. PMID: 31451495; PMCID: PMC6811406.

20. Chen LF, Knelson LP, Gergen MF, Better OM, Nicholson BP, Woods CW, Rutala WA, Weber DJ, Sexton DJ, Anderson DJ; CDC Prevention Epicenters Program. A prospective study of transmission of Multidrug-Resistant Organisms (MDROs) between environmental sites and hospitalized patients-the TransFER study. Infect Control Hosp Epidemiol. 2019 Jan;40(1):47-52. doi: 10.1017/ice.2018.275. Epub 2018 Nov 14. PMID: 30426908; PMCID: PMC6690181.

21. Girija As S, Priyadharsini J V. CLSI based antibiogram profile and the detection of MDR and XDR strains of Acinetobacter baumannii isolated from urine samples. Med J Islam Repub Iran. 2019; 33:3. Published 2019 Feb 8. doi:10.34171/mjiri.33.3

22. Khan F, Khan A, Kazmi SU. Prevalence and Susceptibility Pattern of Multi Drug Resistant Clinical Isolates of Pseudomonas aeruginosa in Karachi. Pakistan Journal of Medical Sciences. 2014 Sep;30(5):951-954. DOI: 10.12669/pjms.305.5400.

23. Alyssa R. Golden, Margot Rosenthal, Ben Fultz, Kimberly A. Nichol, Heather J. Adam, Matthew W. Gilmour, Melanie R. Baxter, Daryl J. Hoban, James A. Karlowsky, George G. Zhanel, Characterization of MDR and XDR Streptococcus pneumoniae in Canada, 2007-13, Journal of Antimicrobial Chemotherapy, Volume 70, Issue 8, August 2015, Pages 21992202, https://doi.org/10.1093/jac/dkv107

24. Basak S, Singh P, Rajurkar M. Multidrug Resistant and Extensively Drug Resistant Bacteria: A Study. J Pathog. 2016; 2016:4065603. doi:10.1155/2016/4065603

\footnotetext{
Cite this Article: Arash Eatemadi, Elham Al Risi, Amit Kasilas Kasliwal, Ahmed Al Za'abi, Hamid Moradzadegan, Zahra Aslani (2021). A Proposed Evidence-Based Local Guideline for Definition of Multidrug-Resistant (MDR), Extensively Drug-Resistant (XDR) and Pan Drug-Resistant (PDR) Bacteria by the Microbiology Laboratory. International Journal of Current Science Research and Review, 4(3), 146-153
} 